\title{
Highlights from the issue
}

\section{Making the best use of blood}

The INTERVAL trial is currently evaluating the effect of reducing the time that must elapse between blood donations by volunteer donors. I've been randomised to donate a unit every 10 weeks so I was pleased to read about the efforts Kelly et al have undertaken in Edinburgh to improve blood component ordering, usage and traceability. There was a $64 \%$ reduction in blood component ordering, a 39\% reduction in blood component transfusion and a $96 \%$ reduction in unaccounted units between 2007 and 2011. That's a lot of armfuls. With increasing pressure on donors and donor services, and increasing awareness of the risks of unnecessary transfusion, there are valuable lessons to be learnt from this study.

\section{When is a head injury not a head injury?}

Decision rules can be used to select patients with head injury for CT scanning, but deciding when to apply the rule can be tricky. If a decision rule is developed on selected patients with evidence of significant trauma, such as loss of consciousness, but then used on every patient with a scalp wound it is unlikely to achieve the anticipated reduction in CT use. Little et al demonstrate this problem by comparing three paediatric head injury decision rules in children with head injury of any severity. The CHALICE rule was applicable to $97 \%$ of the population, whereas the CATCH rule was only applicable to $26 \%$. This makes it difficult to compare the performance of these rules using existing data.

\section{Reducing shoulder dislocation without sedation}

Recruiting acutely injured patients to randomised trials is a huge challenge, so
Josephine Yuen Man Chung et al from Hong Kong should be congratulated, along with their trial participants, for undertaking a randomised trial of the Oxford chair method for reducing anterior shoulder dislocation. This method, described in the paper, allowed reduction without the use of sedation in 23 out 30 patients $(77 \%)$. There were no significant differences in pain score, median procedure time or median length of stay in the emergency department. The remaining seven patients in the intervention group were converted to traditional methods after unsuccessful attempted reduction in the chair.

\section{OK, it's not serious, but what was it?}

Patients like to have a diagnosis. Emergency department investigation is geared towards identifying serious causes but when these are excluded the physician will often have to concede that the diagnosis remains uncertain. We can sometimes come up with a benign diagnosis, such as muscular chest pain or tension headache, but how do we know that these are really the cause without a definitive diagnostic test? It may be more honest to admit we simply don't know. Wen et al explored this issue and found that only $17 \%$ of patients presenting with chest pain, $43 \%$ with abdominal pain and $43 \%$ with headache, the received a pathological discharge diagnosis after emergency department assessment. That leaves a lot of people wondering what caused their symptoms. The most important issue may ultimately be prognosis. Perhaps if we can reassure these patients that they can expect a benign course with spontaneous resolution of their symptoms then they will accept our diagnostic uncertainty.

\section{Antithrombotic agents and head injury}

Increasing numbers of people are receiving antithrombotic agents for a variety of long-term medical conditions. As a result an increasing proportion of head injured patients are taking these agents, but little is known about their effect upon head injury diagnosis and prognosis. Falzon et al studied 345 head injured patients, of whom 164, 70, 55 and 56 were taking aspirin, warfarin, clopidogrel and a combination of agents respectively. Mortality rates during follow-up were high, ranging from $10 \%$ in the warfarin group to $41 \%$ in the combined agent group. This probably reflects the age and high rate of co-morbidities in this cohort. Emergency physicians need to be aware of the high risk of adverse outcome in this group, although whether they can do anything to reduce this risk is another matter.

\section{Safe sex?}

Pfortmueller et al warn us that sexual activity is mechanically dangerous, potentially infectious and stressful for the cardiovascular system. Fortunately they also reassure us that most people enjoy sexual intercourse without complications. Over 11 years they identified 445 patients with an emergency department admission directly related to sexual intercourse. This may represent an underestimate (the lack of foreign bodies in body cavities suggests some under-reporting) but it still seems reassuringly small in comparison to the relevant denominatorthe number of sexual interactions occurring in the local population over the 11 years of the study. 